\title{
Rational Modeling of Multiport Systems by Modal Vector Fitting
}

\author{
Bjørn Gustavsen ${ }^{1}$, and Christoph Heitz ${ }^{2}$ \\ ${ }^{1}$ SINTEF Energy Research, N-7465 Trondheim, Norway \\ ${ }^{2}$ Institut für Datenanalyse und Prozessdesign, CH-8401 Winterthur, Switzerland \\ bjorn.gustavsen@sintef.no, christoph.heitz@zhwin.ch
}

\begin{abstract}
This paper introduces a new approach for rational macromodeling of multiport devices that ensures high accuracy with arbitrary terminal conditions. This is achieved by reformulating the vector fitting technique to fit eigenpairs rather than matrix elements. By choosing the least squares weighting equal to the inverse of the eigenvalue magnitude is achieved that the eigenvalues are fitted with a relative accuracy criterion. The procedure is shown to give a major improvement in accuracy for cases with a large eigenvalue spread. Also is shown how to utilize the impedance characteristics of the adjacent network in the fitting process.
\end{abstract}

\section{Introduction}

Wideband modeling of devices and systems from tabulated data is becoming of major importance for the design and verification of microwave systems. The modeling is usually based on "fitting" a model to a set of parameters that characterize the model behavior, such as $y-, z$-, and $s$ parameters in the frequency domain or the time domain. The fitting process is usually based on a ratio of polynomials $[1],[2]$ or orthogonal polynomial functions [3]. Recently, the pole relocating vector fitting technique [4] has become widely applied, and several enhancements have been proposed [5],[6],[7]. The modeling is complete when the parameters have been fitted to a given accuracy level. However, as a parameter set corresponds to a specific terminal condition, there is no guarantee that the model will behave satisfactorily with a different terminal condition. (For instance, yparameters correspond to a voltage application whereas $z$ parameters correspond to a current application).

In this paper is introduced a more general way of characterizing accuracy by requiring that the model behaves accurately with arbitrary terminal conditions [8]. This is achieved by focusing on the relative accuracy of eigenvalues (modes) rather than matrix elements. This concept is merged with the vector fitting (VF) technique, leading to Modal Vector fitting (MVF). Also is shown how to utilize a priori knowledge about the terminal conditions.

\section{Accuracy considerations}

As an example we consider a system described by its admittance $y$-parameters. The admittance matrix $\mathbf{Y}$ defines the current response $\mathbf{i}$ when applying voltages $\mathbf{v}$ to the ports.

$$
\mathbf{i}(s)=\mathbf{Y}(s) \mathbf{v}(s)
$$

If current sources are applied to the terminals, the voltage response at any frequency is

$$
\mathbf{v}=\mathbf{Z i}=\mathbf{Y}^{-1} \mathbf{i}=\left(\mathbf{T}_{Y} \Lambda \mathbf{T}_{Y}^{-1}\right)^{-1} \mathbf{i}=\mathbf{T}_{Y} \Lambda_{Y}^{-1} \mathbf{T}_{Y}^{-1} \mathbf{i}
$$

where $\Lambda_{Y}$ is a diagonal matrix holding the eigenvalues of $\mathbf{Y}$.
Clearly, small eigenvalues of $\mathbf{Y}$ become large eigenvalues in $\mathbf{Z}$. If $\mathbf{Y}$ contains both large and small eigenvalues, rational fitting of the elements of $\mathbf{Y}$ is likely to result in a poor representation of the small eigenvalues. Thus, fitting the elements of $\mathbf{Y}$ results in a model that is best suited for reproducing terminal currents if the voltages are given. However, the model is not well suited for reproducing voltages with given currents.

It is therefore proposed to fit the model in such a way that the error of the model eigenvalues is related to the eigenvalue magnitude by a relative criterion,

$$
\left|\frac{\lambda_{i}^{\text {model }}(s)-\lambda_{i}^{\text {data }}(s)}{\lambda_{i}^{\text {data }}(s)}\right|<\varepsilon, i=1 \ldots n
$$

Usage of this criterion will make sure that the model behaves accurately (with errors in same order of magnitude) with both voltage application and with current application, and with any other (hybrid) terminal condition.

The general problem considered in this paper is to identify a pole-residue model (4) with $\mathbf{D}$ and $\mathbf{E}$ possibly zero, in such a way that the accuracy of the eigenvalues of $\mathbf{Y}$ is preserved in the relative sense by criterion (3).

$$
\mathbf{Y}(s) \cong \mathbf{Y}_{r a t}(s)=\sum_{m} \frac{\mathbf{R}_{m}}{s-a_{m}}+\mathbf{D}+s \mathbf{E}
$$

\section{Vector Fitting}

Rational fitting of a frequency response $y(s)$ by vector fitting (VF) [4] amounts to solving the linear problem (5) with a set of predefined poles, $\left\{a_{m}\right\}$.

$$
\overbrace{\left(\sum_{m=1}^{N} \frac{\tilde{r}_{m}}{s-a_{m}}+1\right)}^{\sigma(s)} y(s) \cong \sum_{m=1}^{N} \frac{r_{m}}{s-a_{m}}+d+s e
$$

After solving (5), an improved pole set for $y(s)$ is calculated by solving the eigenvalue problem (6) where $\mathbf{A}$ is a diagonal matrix holding the poles $\left\{a_{m}\right\}$, $\mathbf{b}$ is a column of ones, and $\mathbf{c}$ holds the residues $\left\{\tilde{r}_{m}\right\}$.

$$
\left\{a_{m}\right\}=\operatorname{eig}\left(\mathbf{A}-\mathbf{b} \cdot \mathbf{c}^{T}\right)
$$

The new (relocated) poles obtained by (6) are reused in (5) in an iterative procedure. This pole relocation procedure usually converges in a few iterations. In the final step, the residues are calculated by solving (5) with $\sigma(s)=1$.

\section{Modal Vector Fitting}

We consider a multi-port device that is characterized by its admittance matrix, Y. This matrix is diagonalized by a (frequency dependent) transformation matrix $\mathbf{T}$ and is to be approximated by a rational model with behavior $\mathbf{Y}_{\text {rat }}$. 


$$
\mathbf{Y}=\mathbf{T} \cdot \boldsymbol{\Lambda} \cdot \mathbf{T}^{-1} \cong \mathbf{Y}_{r a t}
$$

Postmultiplying (7) with $\mathbf{T}$ gives for each eigenpair $\left(\lambda_{i}, \mathbf{t}_{i}\right)$

$$
\mathbf{Y}_{r a t} \cdot \mathbf{t}_{i} \cong \lambda_{i} \cdot \mathbf{t}_{i}
$$

The relative accuracy of the eigenvalue is retained in the least squares problem by scaling the equation with the inverse of the eigenvalue magnitude (9). It is remarked that this scaling is a frequency dependent quantity.

$$
\frac{1}{\left|\lambda_{i}\right|}\left(\mathbf{Y}_{\text {rat }} \cdot \mathbf{t}_{i}-\lambda_{i} \cdot \mathbf{t}_{i}\right) \cong 0, i=1, . ., n
$$

Combining (9) with VF leads to Modal VF (MVF). For the pole identification step we get

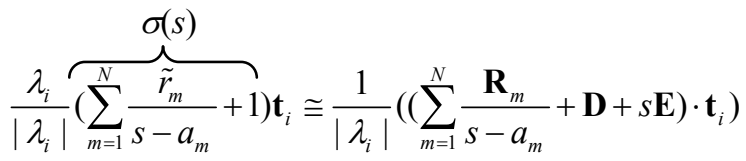

Equation (10) is built for all modes $i=1 . . n$ and stacked into a common equation. The new (relocated) poles are obtained from $\sigma(s)$ in (10) by (6), as in the original VF. Finally, the residues are calculated by solving (10) with $\sigma(s)=1$.

\section{Utilizing external circuit properties}

In some situations the considered device is to be connected to an external network with know impedance characteristics, see Fig. 1. If the admittance seen from the terminals of the device is $\mathbf{Y}_{e x t}$, the total admittance is

$$
\mathbf{Y}_{\text {tot }}=\mathbf{Y}_{\text {device }}+\mathbf{Y}_{\text {ext }}
$$

The terminal behavior is now governed by $\mathbf{Y}_{\text {tot }}$ rather than $\mathbf{Y}_{\text {device. }}$. This can be utilized in the MVF fitting process by calculating eigenpairs from $\mathbf{Y}_{\text {tot }}$. Equation (10) now becomes

$$
\begin{aligned}
& \frac{\lambda_{i}}{\left|\lambda_{i}\right|} \overbrace{\left(\sum_{m=1}^{N} \frac{\tilde{r}_{m}}{s-a_{m}}+1\right) \mathbf{t}_{i}}^{\sigma(s)} \\
& \cong \frac{1}{\left|\lambda_{i}\right|}\left(\left(\mathbf{Y}_{\text {ext }}+\sum_{m=1}^{N} \frac{\mathbf{R}_{m}}{s-a_{m}}+\mathbf{D}+s \mathbf{E}\right) \cdot \mathbf{t}_{i}\right)
\end{aligned}
$$

The final computation of residues (with known poles) is done with $\sigma(s)$ in (12) equal to unity.

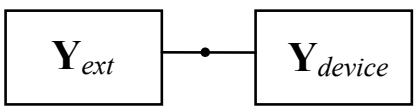

Fig. 1 External network

\section{Example}

As an example is used a lossy conductor over a lossy earth of $5 \mathrm{~km}$ length, see Fig. 2. This gives a $2 \times 2 \mathbf{Y}$.

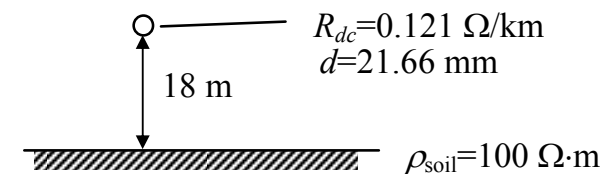

Fig. 2 Single conductor overhead line
A pole-residue model for $\mathbf{Y}$ was calculated in the frequency range $1 \mathrm{~Hz}-100 \mathrm{kHz}$ using $\mathrm{VF}$ and MVF. In both cases, the fitting process used five iterations with 14 poles and a nonzero $\mathbf{D}$.

Fig. 3 shows the eigenvalues of $\mathbf{Y}$. It is observed that when the eigenvalue spread is large (low frequencies), the small eigenvalue becomes inaccurately represented when fitting $\mathbf{Y}$ by VF. With MVF, all eigenvalues are accurately represented at all frequencies, due to the relative criterion (3).

Fig. 4 (solid traces) shows the elements of $\mathbf{Y}$. In the same plot is shown the deviation by the rational models from the correct solution is shown in the same plot. It is observed that with MVF, the deviation curves are closely correlated with the element magnitude, while VF gives "flatter" deviation curves. Otherwise, the quality of the fittings appear similar.

Fig. 5 shows the result for $\mathbf{Z}=\mathbf{Y}^{-1}$, which corresponds to the voltage response when applying currents to the line ends. It is seen that the result by MVF remains accurate while that by VF is poor. The latter result is caused by the inability of VF to accurately represent the small eigenvalues, as was shown in Fig. 3. Since the small eigenvalues of $\mathbf{Y}$ become the large eigenvalues of $\mathbf{Z}$ (2), a catastrophic error magnification takes place. With MVF, the relative accuracy of eigenvalues is preserved and so an accurate result is ensured also for $\mathbf{Z}$.

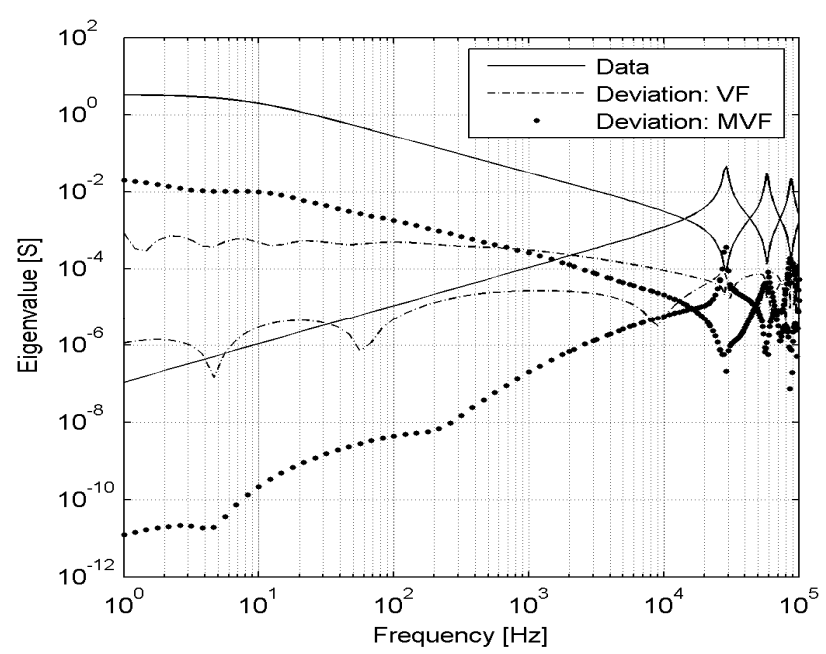

Fig. 3 Eigenvalues of $\mathbf{Y}$

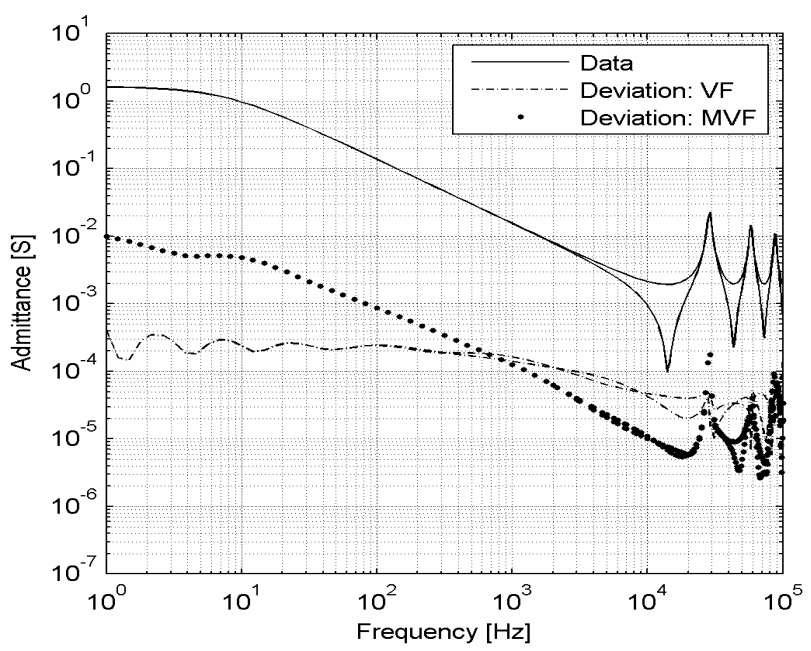

Fig. 4. Elements of $\mathbf{Y}$ 


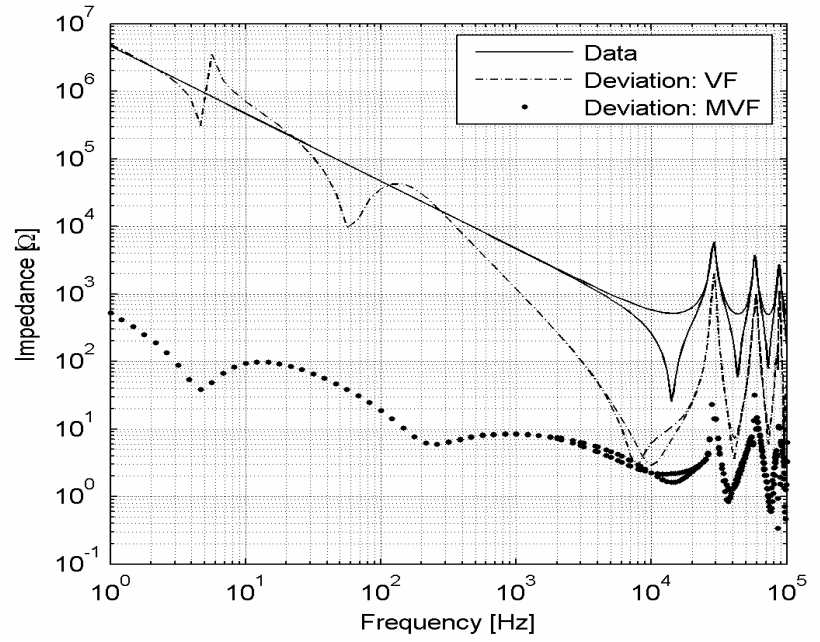

Fig 5. Elements of $\mathbf{Z}=\mathbf{Y}^{-1}$

Fig. 6 shows the sparsity pattern of the system matrix for the pole identification step. When utilizing symmetry, usage of VF leads to the simultaneous fitting of 3 elements, giving 3 blocks of size $14+1$ on the diagonal (left panel). In addition comes the contribution from the sigma function which adds another 14 unknowns. With MVF, a less sparse matrix results.
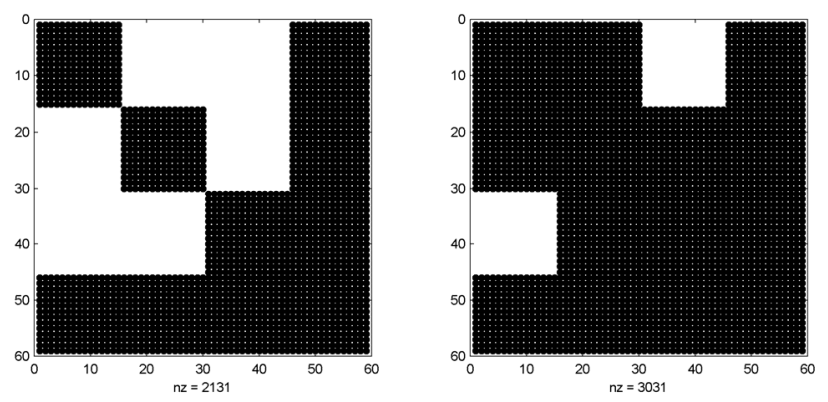

Fig. 6 Sparsity pattern: VF (left) and MVF (right)

\section{Result with inclusion of external network}

We proceed with the same example but assume that the line is terminated at both ends with a $1 \mathrm{k} \Omega$ resistor, see Fig. 7 . The fitting of $\mathbf{Y}_{\text {device }}$ by MVF is now done using (12).

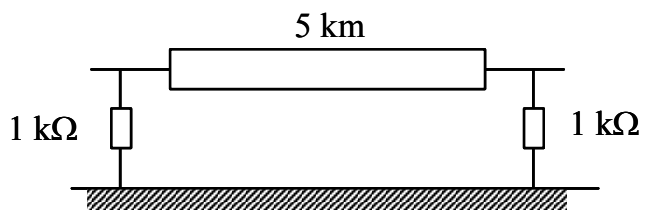

Fig. 7 Connecting Transmission line to external network

The eigenvalues of $\mathbf{Y}_{\text {device }}+\mathbf{Y}_{\text {ext }}$ are shown in Fig. 8. The eigenvalue spread is much smaller than in Fig. 3 due to the connection to ground by the $1 \mathrm{k} \Omega$ resistors. Usage of $\mathrm{VF}$ and MVF appear to give a similar result. However, inspection of deviation curves shows that the result by MVF is more accurate for the small eigenvalue, see Fig. 9.

As in the previous example, both the VF and MVF approaches give a satisfactory result for the fitted elements of $\mathbf{Y}_{\text {device }}$ (Fig. 10). But for the elements of $\mathbf{Z}=\left(\mathbf{Y}_{\text {device }}+\mathbf{Y}_{\text {ext }}\right)^{-1}$ (Fig. 11), MVF gives a more accurate result due to the better representation of the small eigenvalue.

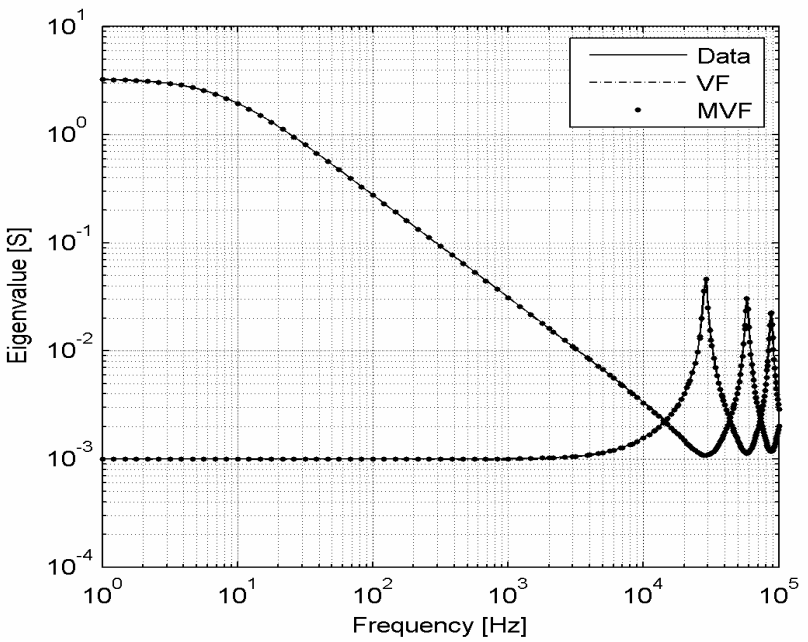

Fig 8 Eigenvalues of $\mathbf{Y}_{\text {tot }}=\mathbf{Y}_{\text {device }}+\mathbf{Y}_{\text {ext }}$

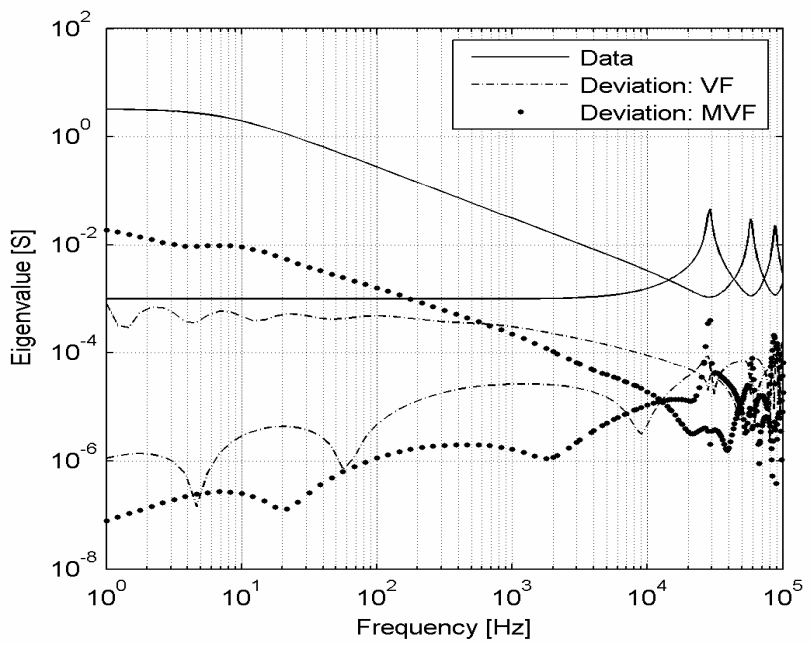

Fig 9 Eigenvalues of $\mathbf{Y}_{t o t}=\mathbf{Y}_{\text {device }}+\mathbf{Y}_{\text {ext. }}$. Deviation curves

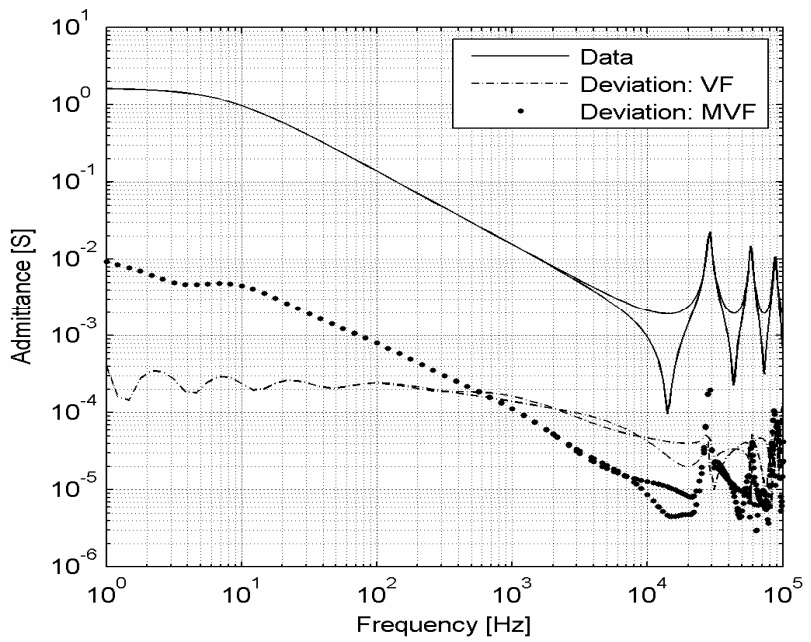

Fig. 10 Elements of $\mathbf{Y}_{\text {device }}$ 


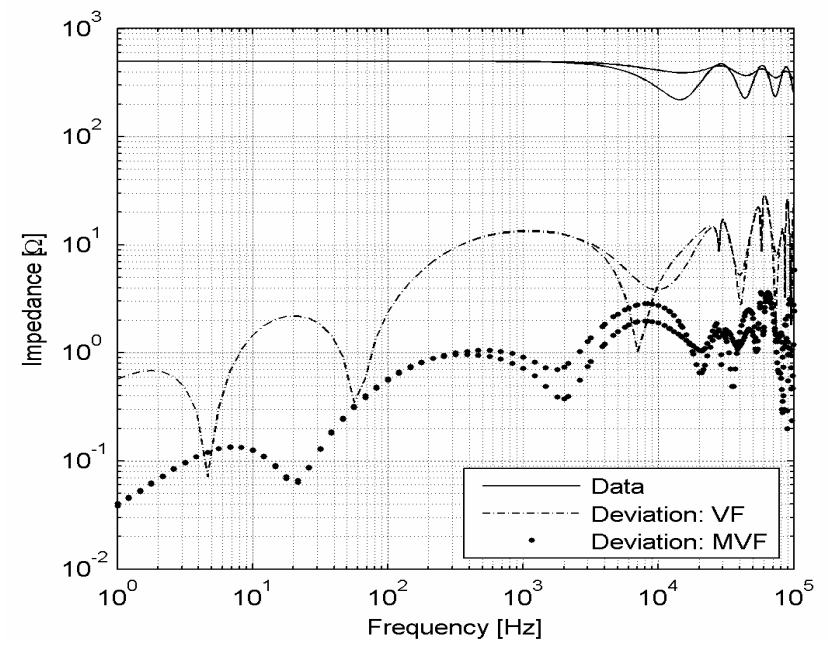

Fig 11 Elements of $\mathbf{Z}=\left(\mathbf{Y}_{\text {device }}+\mathbf{Y}_{\text {ext }}\right)^{-1}$

\section{Discussion}

In some situations, a constant, real transformation matrix $\mathbf{T}_{Y}$ can be assumed, for instance when $\mathbf{Y}$ is a balanced matrix (which was the case for the example in this paper). This allows to diagonalize $\mathbf{Y}$ and fit the eigenvalues directly. Usage of inverse magnitude weighting then gives a result similar to MVF. However, in many cases the assumption of a constant $\mathbf{T}_{Y}$ does not apply and so MVF must be used.

In a direct application of VF to the matrix elements, one can of course increase the fitting order while monitoring the eigenvalues of $\mathbf{Y}_{\text {model }}$ vs. $\mathbf{Y}_{\text {device}}$. This can give an equally good result as MVF, at the cost of a higher fitting order.

A different situation is when modeling from noisy measurements. In [8],[9] was proposed to measure a set of voltage/current vector pairs that correspond to the system eigenpairs. Combined with rational fitting and passivity enforcement, this has led to the development of the SoFT tool [10]. Usage of MVF is here advantageous since the noise level is usually much lower for eigenpairs corresponding to small eigenvalues. Direct application of VF to matrix elements can easily result in that the smallest eigenvalues are lost in the noise. Also, since the SoFT measurement approach does not assume a constant transformation matrix, direct fitting of eigenvalues does not apply. This application was the main motivation for developing MVF.

In practical applications, the device may have a large eigenvalue spread while at the same time it is connected to an external network such that the eigenvalue spread of the combined network as seen from the terminals is greatly reduced. If this knowledge is not utilized, an unnecessary constrained fitting will result. This problem is easily avoided by considering the external network when calculating eigenpairs as was demonstrated in this paper.

The idea of preserving the relative accuracy of small eigenvalues also applies when enforcing passivity by perturbation. In [11],[12] is described how to achieve this by modifying the passivity enforcement approach in [13].

\section{Conclusions}

A reformulated vector fitting (MVF) has been developed that ensures high accuracy of the obtained model with arbitrary terminal conditions. This is achieved by explicitly introducing eigenpairs in the modeling, thereby allowing to ensure high relative accuracy for all eigenvalues. Application to an example with a large eigenvalue spread demonstrated superior accuracy over the traditional approach of fitting matrix elements. The MVF approach also allows to take into account the impedance characteristics of the adjacent network.

\section{References}

[1] E.C. Levy, "Complex curve fitting", IRE Trans. Automatic Control, vol. 4, pp. 37-44, May 1959.

[2] C.K. Sanathanan and J. Koerner, "Transfer function synthesis as a ratio of two complex polynomials", IEEE Trans. Automatic Control, vol. 8, pp. 56-58, 1963.

[3] C.P. Coelho, J.R. Phillips, and L.M. Silveira, "Generating high-accuracy simulation models using problem-tailored orthogonal polynomials basis", IEEE Trans. Circuits and Systems - I, vol. 53, no. 12, pp. 2705-2714, Dec. 2006.

[4] B. Gustavsen, and A. Semlyen, "Rational approximation of frequency domain responses by vector fitting", IEEE Trans. Power Delivery, vol. 14, no. 3, pp. 1052-1061, July 1999.

[5] S. Grivet-Talocia, "Package macromodeling via timedomain vector fitting", IEEE Microwave and Wireless Components Letters, vol. 13, no. 11, pp. 472 474, Nov. 2003.

[6] B. Gustavsen, "Improving the pole relocating properties of vector fitting”, IEEE Trans. Power Delivery, vol. 21, no. 3, pp. 1587-1592, July 2006.

[7] D. Deschrijver, B. Haegeman, and T. Dhaene, "Orthonormal vector fitting: A robust macromodeling tool for rational approximation of frequency domain responses", IEEE Trans. Advanced Packaging, accepted.

[8] C. Heitz, and A. Steiner, "Algorithm for an automated modal model generation from measured data for electrical N-ports", unpublished ABB report, Dec. 2002.

[9] K. Niayesh, M. Berth, A. Dahlquist, and C. Heitz, "Measurement algorithm for optimal characterization of multi-port electrical components and systems", unpublished ABB invention disclosure, Dec. 2002.

[10]M. Tiberg, O. Hoenecke, C. Heitz, and B. Gustavsen, "New simulation methodology for power systems assumption free analysis entirely based on measurement", EMTP-RV Newsletter, vol. 1, no 3, Dec. 2006, pp. 20-26.

[11]B. Gustavsen, "Passivity enforcement of rational models via modal perturbation", IEEE Trans. Power Delivery, submitted.

[12]B. Gustavsen, "Fast passivity enforcement of rational macromodels by perturbation of residue matrix eigenvalues", Proc. IEEE Workshop on Signal Propagation on Interconnects, submitted.

[13]B. Gustavsen, and A. Semlyen, "Enforcing passivity for admittance matrices approximated by rational functions", IEEE Trans. Power Systems, vol. 16, no. 1, pp. 97-104, Feb. 2001. 